\title{
EELSpecNet: Deep Convolutional Neural Network Solution for Electron Energy Loss Spectroscopy Deconvolution
}

S. Shayan Mousavi M. ${ }^{1}$, Alexandre Pofelski ${ }^{1}$ and Gianluigi Botton ${ }^{2}$

${ }^{1}$ McMaster University, Canada, ${ }^{2}$ Department of Materials Science and Engineering, McMaster University, Hamilton, ON, Canada, Canada

Electron energy loss spectroscopy (EELS) is a powerful technique for studying the chemical and electronic properties of materials at the nanometer length-scale. Recent technological advancements have made nearmeV energy-resolution spectroscopy more accessible than ever (1). Near meV-resolution spectroscopy is usually implemented for purposes such as exploring plasmonic and phononic activities and measuring energy gains. However, similar to any other microscopy techniques, the output spectra usually suffer from the presence of high frequency (HF) noise and are convoluted with the optical transfer function (OTF), also known as the point spread function (PSF). Spectral deconvolution is of major importance to reveal data hidden under the broad tails of the zero-loss peak. The most frequently used approaches for spectral deconvolutions are Filter-based methods and partial differential equation (PDE) fitting methods (2-5). In the case of PDE fitting methods, comprehensive prior knowledge about the HF noise, OTF, and the physical phenomena that are being probed is required, thus, implementation of these methods is not usually a simple task. Moreover, making assumptions for different signal modulations for this technique can drastically affect the deconvolution process and the outcome. PDE methods requiring vast background information are considered non-blind techniques. On the other hand, filter-based methods are semi-blind methods and simple to implement but are usually amplifying the high-frequency noise and/or adding artifacts. For example, the Richardson-Lucy (RL) and the Maximum Likelihood (ML) methods are widely applied filter-based iterative methods using a captured PSF and assuming a Poisson distribution noise $(6,7)$. A common artifact from both methods is to introduce high-frequency components in the deconvolved signal when high iterations of the deconvolution process are used. In recent years, deep learning algorithms have found various applications in the field of electron microscopy including our prior work on valence state identification and mapping (8). In this work a blind deconvolutional neural network architecture, EELSpecNet, is presented to tackle current challenges in EELS spectral deconvolution. EELSpecNet is a python script inspired by the U-shaped and dilated deep neural network architectures $(5,9)$. The outcome of the deep learning deconvolution approach is presented and compared with RL and Fourier Transform methods for deconvolving low-loss EELS spectra. For training the network, an artificially convoluted spectral dataset is used. For this purpose, principal component analysis (PCA) is applied for separating components in experimentally captured low-loss EELS spectra. Subsequently, spectra generated with low-frequency signals are set as the reference spectra, and high-frequency components and experimentally captured PSF are used for creating the artificially convoluted dataset. The advantage of EELSpecNet is to not use any pre-existing knowledge (such as the PSF) and any assumption on the noise distribution. Nevertheless, the training process should be closely monitored and can be computationally expensive. Although EELSpecNet is intended to be used for low-loss EELS spectral deconvolution, the same method can be extended to other spectral deconvolution (not limited to EELS), feature classifications (e.g., peaks, edges, background, and fine structures), and segmentation with minor modifications and a dedicated training set (10). 


\section{References}

1. Krivanek OL, Lovejoy TC, Dellby N, Aoki T, Carpenter RW, Rez P, et al. Vibrational spectroscopy in the electron microscope. Nature. 2014 Oct;514(7521):209-12.

2. Egerton RF, Williams BG, Sparrow TG. Fourier deconvolution of electron energy-loss spectra. Proceedings of the Royal Society of London A Mathematical and Physical Sciences. 1985;398(1815):395-404.

3. Wang F, Egerton R, Malac M. Fourier-ratio deconvolution techniques for electron energy-loss spectroscopy (EELS). Ultramicroscopy. 2009;109(10):1245-9.

4. Bellido EP, Rossouw D, Botton GA. Toward $10 \mathrm{meV}$ electron energy-loss spectroscopy resolution for plasmonics. Microsc Microanal. 2014;20(3):767-78.

5. Zhu H, Qiao Y, Xu G, Deng L, Yu Y-F. Dspnet: A lightweight dilated convolution neural networks for spectral deconvolution with self-paced learning. IEEE Transactions on Industrial Informatics. 2019;16(12):7392-401.

6. Richardson WH. Bayesian-based iterative method of image restoration. JoSA. 1972;62(1):55-9.

7. Lucy LB. An iterative technique for the rectification of observed distributions. The astronomical journal. 1974; 79:745.

8. Chatzidakis M, Botton GA. Towards calibration-invariant spectroscopy using deep learning. Scientific reports. 2019;9(1):1-10.

9. Falk T, Mai D, Bensch R, Çiçek Ö, Abdulkadir A, Marrakchi Y, et al. U-Net: deep learning for cell counting, detection, and morphometry. Nature Methods. 2019 Jan;16(1):67-70.

10. We are grateful to the Natural Sciences and Engineering Research Council for supporting this work. 